Association for Information Systems AIS Electronic Library (AISeL)

2018 Proceedings

Portugal (CAPSI)

2018

\title{
Using kahoot as a learning tool
}

Ernane Rosa Martins

Instituto Federal de Educação, Ciência e Tecnologia de Goiás (IFG), ernane.martins@ifg.edu.br

Wendell Bento Geraldes

Instituto Federal de Educação, Ciência e Tecnologia de Goiás (IFG), wendell.geraldes@ifg.edu.br

Ulisses Rodrigues Afonseca

Instituto Federal de Educação, Ciência e Tecnologia de Goiás (IFG), urafonseca@ifg.edu.br

Luís Manuel Borges Gouveia

Universidade Fernando Pessoa, lmbg@ufp.edu.pt

Follow this and additional works at: https://aisel.aisnet.org/capsi2018

\section{Recommended Citation}

Martins, Ernane Rosa; Geraldes, Wendell Bento; Afonseca, Ulisses Rodrigues; and Gouveia, Luís Manuel Borges, "Using kahoot as a learning tool" (2018). 2018 Proceedings. 44.

https://aisel.aisnet.org/capsi2018/44

This material is brought to you by the Portugal (CAPSI) at AIS Electronic Library (AISeL). It has been accepted for inclusion in 2018 Proceedings by an authorized administrator of AIS Electronic Library (AISeL). For more information, please contact elibrary@aisnet.org. 


\title{
Uso do kahoot como ferramenta de aprendizagem
}

\author{
Using kahoot as a learning tool
}

Ernane Rosa Martins, Instituto Federal de Educação, Ciência e Tecnologia de Goiás (IFG), Brasil, ernane.martins@ifg.edu.br

Wendell Bento Geraldes, Instituto Federal de Educação, Ciência e Tecnologia de Goiás (IFG), Brasil,wendell.geraldes@ifg.edu.br

Ulisses Rodrigues Afonseca, Instituto Federal de Educação, Ciência e Tecnologia de Goiás (IFG), Brasil, urafonseca@ifg.edu.br

Luís Manuel Borges Gouveia, Universidade Fernando Pessoa, Portugal, lmbg@ufp.edu.pt

\section{Resumo}

O presente trabalho tem como objetivo investigar o uso do "kahoot" como recurso educacional no processo de ensino-aprendizagem para estudantes do ensino médio da área de informática. Para isso, utilizou-se uma abordagem exploratória, descritiva e bibliográfica. A pesquisa foi realizada com 34 alunos do curso técnico em informática para internet do Instituto Federal de Goiás (IFG). Os alunos realizaram as atividades propostas e em seguida responderam a um questionário através de um formulário do GoogleDocs. De acordo com os resultados da utilização da plataforma, verificou-se uma melhora na aprendizagem dos alunos revisando e reforçando os conceitos aprendidos de maneira divertida, engajadora, motivadora e interessante.

Palavras-chave: Kahoot; Gamificação; Ensino; Tecnologia Educacional; Aprendizagem Móvel.

\begin{abstract}
The present work aims to investigate the use of kahoot as an educational resource in the teaching-learning process for high school students in the area of computer science. For this, an exploratory, descriptive and bibliographic approach was used. The research was carried out with 34 students of the technical course in computer science for internet of the Federal Institute of Goiás (FIG). The students performed the proposed activities and then answered a questionnaire using a GoogleDocs form. According to the results of the use of the platform, there was an improvement in students' learning by reviewing and reinforcing the concepts learned in a fun, engaging, motivating and interesting way.
\end{abstract}

Keywords: Kahoot; Gamification; Teaching; Educational technology; Mobile Learning.

\section{INTRODUÇÃ̃o}

Os jogos estão presentes na vida das pessoas de todas as idades. As criança aprendem desde cedo a brincar com jogos o que é muito importante por permitir o desenvolvimento de suas habilidades. $\mathrm{O}$ uso dos Jogos em ambientes escolares tem se tornado uma excelente estratégia de ensino. Macedo (2000), afirma que a utilização de jogos no ensino permite melhorar a capacidade de adquirir 
conhecimentos e conteudos abstratos, desenvolver habilidades, motivar a aprendizagem, estimular o raciocinio e a compreender regras. O mesmo autor destaca ainda que a escolha do jogo para utilizar em uma sala de aula é de grande importância, principalmente definindo os objetivos da utilização, para não perder o foco do conteúdo e da aprendizagem.

O jogo é uma estratégia didática, e para se obter um resultado positivo precisa de um conjunto de estratégias. Apenas o ato de jogar não é o suficiente para atingir os objetivos educacionais esperados pelo professor. Alguns fatores devem ser levados em consideração na escolha de uma tecnica de ensino, tais como: as diferenças entre os alunos (idade, contexto sociocultural, aptidões; interesses e necessidades dos alunos); quem a emprega; quais os objetivos a alcançar; quais os tipos de pessoas a que se destina; qual o conteúdo será tratado; em qual momento a técnica será aplicada. O que vem se demonstrando na literatura, é que bons resultados dependem do planejamento do professor e da condução do mesmo na sala de aula. Os jogos devem explorar a possibilidade de combinar entretenimento com educação, desta maneira o conhecimento e o aprendizado se tornam mais motivador. (Covos, et. al., 2018).

O kahoot é um software interativo que contêm elementos dos jogos e permite aplicar uma atividade gamificada com conteúdo específico (Gazotti-Vallim, 2017). O conteúdo é adicionado pelo professor e aplicado em classe, necessitando simplesmente de um computador, internet e dos smartphones dos proprios alunos (Bottentuit Junior, 2012).

A utilização do kahoot em sala de aula tem como finalidade permitir ensinar os alunos a aprender com mais prazer, a construir seus proprios conhecimentos e promover uma melhor comunicação, assim este estudo é inportante por buscar minimizar as dúvidas dos profissionais da educação em utilizar esta ferramenta educacional disponivel, visto o novo perfil digital dos alunos, permitindo que o ensino seja mais bem transmitido, melhor absorvido pelos alunos, menos cansativo e com maior concentração.

Sendo assim, este trabalho propôs investigar o uso do "kahoot" como recurso educacional no processo de ensino-aprendizagem para estudantes do ensino médio da área de informática. Utilizando uma abordagem exploratória, descritiva e bibliográfica.

Este artigo está estruturado em cinco seções. Nesta presente seção apresenta, além da introdução, a definição da problemática de pesquisa, o objetivo, a justificativa e importância do estudo e a estrutura da presente pesquisa. A seção 2 traz o referencial teórico, com a formação de uma base conceitual e teórica, que fornecem subsídios para o desenvolvimento deste estudo. Na seção 3 são apresentados o método empregado e as técnicas e procedimentos metodológicos utilizados. Na seção 4 estão descritos os resultados obtidos na pesquisa e discussão. Por fim, a seção 5 apresenta a conclusão e propõe a continuidade da pesquisa. 


\section{REFERENCIAL TEÓRICO}

Os educadores tem como desafio atualmente a busca por ferramentas didáticas que possibilitem ser utilizadas dentro da sala de aula como metodologias interativas, que torna o ambiente educativo cada vez mais digital (Prensky, 2012). Gómez (2015, p. 29) reforça que "é preciso reinventar a escola para que esta possa desenvolver conhecimentos, habilidades, atitudes, valores e emoções". Sendo assim, o Kahoot é plataforma que pode ser utilizado no ambiente educacional como uma proposta pedagógica, que permiti uma aula com diferentes abordagens e novas possibilidades de avaliação. O Kahoot permite aos alunos aprenderem utilizando um dispositivo tecnológico (computador ou smartphone) enquanto se divertem. Assim, os alunos jogam como se estivessem participando de um game show, o que lhes permite aprender de forma divertida, lúdica e interativa, construindo, seu próprio conhecimento (Kenski, 2012).

O Kahoot é uma plataforma de aprendizagem gratuita baseada em jogos que tem como missão institucional "desbloquear o potencial mais profundo de cada aluno de todas as idades e em todos os contextos" através de um aprendizado divertido, mágico, inclusivo e envolvente (Kahoot, 2018). Esta plataforma permite criar questionários, discussões ou pesquisas que podem ser respondidas por usuários que estejam conectados à internet por meio de smartphones ou computadores. Para sua utilização, faz necessário a realização de um cadastro na plataforma de ensino virtual (https://kahoot.com/).

A proposta do Kahoot é envolver os alunos por meio de questionários, discussões e pesquisas préelaboradas semelhantes a jogos, com pontuação, interação e ranqueamento, (Dellos, 2015). O professor pode usar o conteúdo de uma disciplina, bem como avaliar o desempenho do aluno. Segundo seus criadores, o seu objetivo, é ser uma plataforma baseada em jogo tornando a aprendizagem divertida (Izeki et. al., 2016). Amico et. al., 2017, enfatiza que esta é uma importante ferramenta no auxílio à tecnologia educativa.

No Kahoot! os alunos acessam com um apelido que aparece na tela, permitindo a identificação dos grupos que estão jogando. Deste modo, os alunos não são expostos para o restante da turma. $\mathrm{O}$ professor também configura qual o tempo para responder cada questão, podendo ter perguntas com tempo de respostas diferentes, conforme a dificuldade da pergunta. Por ser similar a um jogo, o tempo não deve ser muito longo, senão a atividade pode perder sua capacidade lúdica. Mas também não pode ser muito longo, pois, não permitiria que o aluno aplicasse o seu conhecimento na resolução.

Os alunos não precisam ter uma conta para utilizar o Kahoot Para entrar, eles devem inserir um pin e o apelido. Ao iniciar, as perguntas junto com as respostas são mostradas na tela grande, e os alunos pressionam a mesma cor e símbolo com a resposta que eles acreditam ser a correta na tela do celular. É exibido um cronômetro, que vai diminuindo até zero, assim como o número de alunos que vão 
respondendo às perguntas. Ao mesmo tempo, os alunos recebem feedback individual sobre como responderam em seus dispositivos. As respostas dos alunos fornecem ao professor feedback sobre a compreensão dos alunos sobre a questão, e cria uma oportunidade para discussão sobre a questão e as respostas. O placar dos cinco melhores, com os pontos e apelidos, é mostrado entre as perguntas. Cada aluno também pode seguir sua própria pontuação e ranking no próprio dispositivo móvel. Para obter uma pontuação alta, os alunos precisam responder corretamente e rapidamente as perguntas. Música e efeitos sonoros são usados no Kahoot para criar suspense e atmosfera de um game show (Wang, 2015). A Figura 1 ilustra como os alunos fornecem suas respostas por meio de dispositivos móveis no Kahoot.

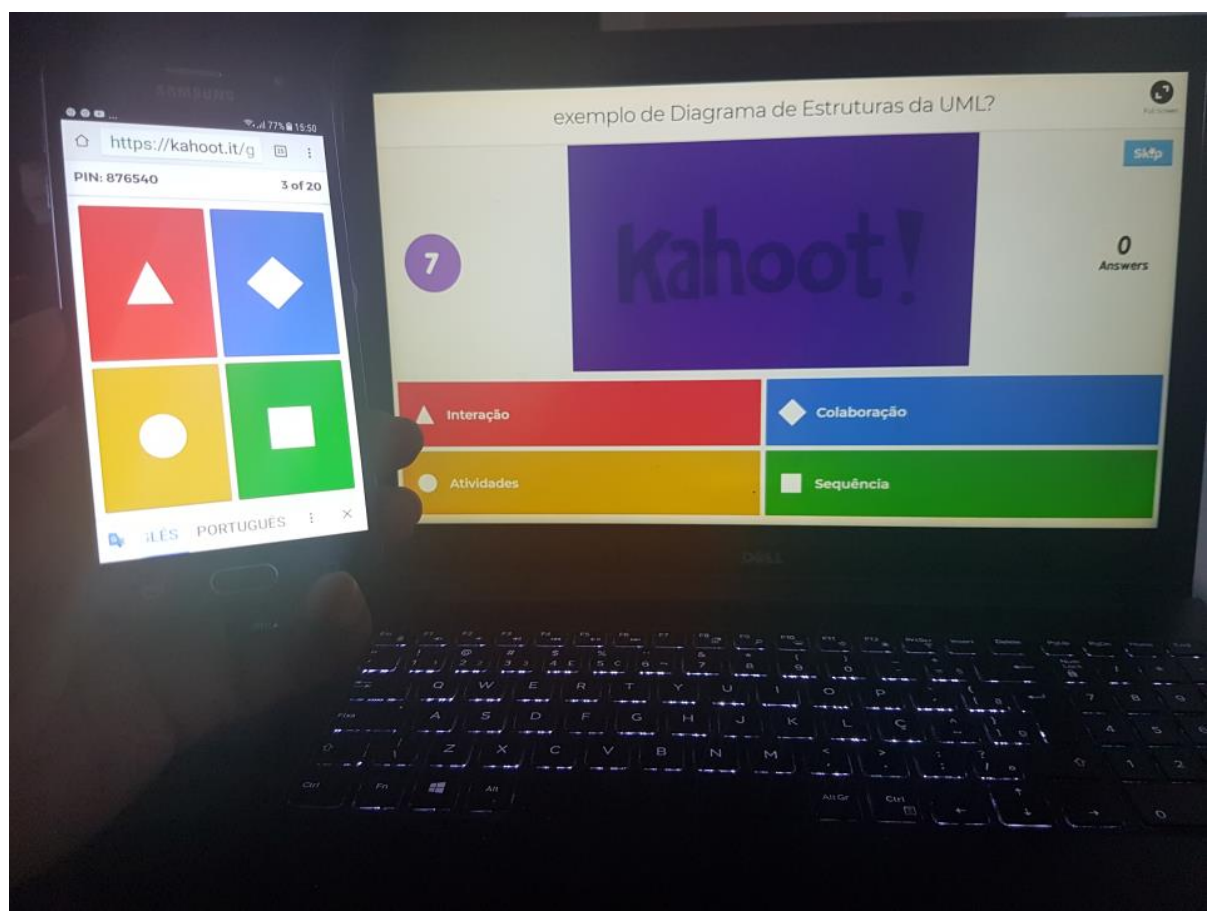

Figura 1 - Como os alunos fornecem as respostas no Kahoot

A ideia principal do Kahoot é ser uma plataforma onde o professor e o os alunos podem interagir em sala de aula simulando um jogo de conhecimento competitivo. A motivação é envolver os alunos através da transformação da sala de aula em um game show, onde o professor seria o apresentador e todos os alunos podem competir ganhando pontos através de respostas corretas em várias questões relacionadas ao assunto que está sendo ensinado na aula (Wang, 2015).

\section{Metodologia de Pesquisa}

A pesquisa tem caráter exploratória, descritiva e bibliográfica. A pesquisa exploratória "tende a abordar novos problemas sobre os quais pouca ou nenhuma pesquisa anterior foi feita" (Brown, 2006). Além disso, deve notar-se que "Pesquisa exploratória é a pesquisa inicial, que forma a base de pesquisas mais conclusivas.” (Singh, 2007). Esta pesquisa foi fundamentada na gamificação na 
educação com o Kahoot como uma atividade interativa em sala de aula. Este estudo exploratório foi implementado em três etapas: Criação, aplicação e avaliação da plataforma.

A primeira etapa consistiu em preparar o quiz on line utilizando o site https://kahoot.com/, antes da aula, pelo professor, para a disciplina de Programação Web do curso técnico em informática para a internet do Instituto Federal de Goiás (IFG) Câmpus Luziânia. A princípio realizou-se o cadastro conforme as informações do site. Em seguida selecionou-se a modalidade "quiz" do Kahoot e as instruções de preenchimento do site para a criação do quiz.

O quiz foi criado com 25 questões de múltipla escolha, com questões de 3 alternativas incorretas e uma correta, todas abordavam o conteúdo ministrado na disciplina. Na criação também foi definido o tempo de 30 segundos para a resposta de cada questão.

No dia da atividade, o professor abriu o quiz on line e disponibilizou o link e o código de acesso para os alunos. Estes estavam familiarizados com a metodologia, pois outro quis já havia sido utilizado pelos alunos anteriormente para apresentar a ferramenta e metodologia. A turma possuía trinta e três alunos que foram divididos em grupos de três, a divisão dos grupos ficou a critério dos próprios estudantes. O quiz foi jogado em grupo, sendo que os mesmos podiam discutir entre eles a melhor resposta.

O plataforma transcorre com a aparição de quatro telas para cada pergunta: primeiro uma tela com a pergunta, a segunda tela com a pergunta, as alternativas, e a contagem do tempo, a terceira tela com a marcação da resposta certa e com as frequências de acertos e erros daquela pergunta e a quarta tela com a classificação parcial do teste até o presente momento. Após a última pergunta, aparece um pódio com a classificação dos três primeiros lugares.

Após a atividade, os alunos foram convidados a responder um questionário on line construído usando um formulário do GoogleDocs. O link foi compartilhado com os alunos por meio de um grupo da turma no WhatsApp, que foi criado pelo professor, para a disciplina, com o propósito de facilitar a interação entre alunos e professor, compartilhar informações e materiais de estudo. Eles responderam o formulário em sala, individualmente. O formulário contava com cinco perguntas, sendo elas: Quais as limitações do uso da tecnologia móvel no processo de aprendizagem? Como o uso do aplicativo durante a atividade contribuiu para a sua aprendizagem? Quais os pontos positivos na utilização de tecnologias móveis no processo de aprendizagem? Quais os pontos negativos na utilização de tecnologias móveis no processo de aprendizagem? Quais as suas sugestões em relação a atividade?

\section{Resultados E DiscuSsão}

Como principais limitações do uso da tecnologia móvel no processo de aprendizagem, tivemos: a baixa velocidade da internet disponivel pela instituição, alguns dos aparelhos dos alunos, o tamanho 
reduzido da tela, a possiblidade de acessar outros conteúdos que não tem relação com a aula durante a atividade proposta.

Ao perguntar como o uso do aplicativo durante a atividade contribuiu para a sua aprendizagem? Eles responderam: a troca de conhecimento por meio dos debates, deixou a aula e o conteúdo mais interessantes, aumentou a concentração e envolvimento dos alunos, tornou o aprendizado mais vivencial, divertida, e interativa, estimulou o entenrece do aluno, aumentou a velocidade de raciocínio para responder em menor tempo e corretamente, e permitiu o trabalho em grupo.

Quando solicitado para apontar quais os pontos positivos na utilização de tecnologias móveis no processo de aprendizagem? Disseram: incentivou todos a participarem da atividade de maneira mais dinâmica e moderna, estimulou a concentração, tornou o aprendizado mais divertido, incitou uma concorrência saudável entre os alunos, despertou o interesse dos alunos nas aulas, melhorou a interação entre os alunos e professor e entre os alunos, e facilitou a aprendizagem.

Entre tanto, em relação aos pontos negativos na utilização de tecnologias móveis no processo de aprendizagem? Responderam: possibilidade de poder tirar o foco da aprendizagem e usar o celular para outras coisa, o tamanho da tela e as conexões de internet lentas, a desigualdade de aparelhos entre os colegas pode gera uma certa desvantagem por alguns, ou se algum estudante não possuir aparelho ou acesso a internet.

Quando solicitados a fornecer sugestões em relação a atividade? Apresentaram: mais atividades como essa durante o semestre, novas questões para repetição do tema., manter o aplicativo e, se possível, aderir a outros similares, utilizar de forma individual, maior tempo para responder.

Grande parte das respostas dos alunos confirmaram Dellos (2015) que afirma que o Kahoot é um plataforma que possibilita maior interação entre os alunos, envolvendo e encorajando eles a não desistirem facilmente das dificuldades encontradas nas disciplinas. A solicitação pela maioria dos alunos em continuar a utilizar o Kahoot nas aulas confirma Sandy e Sandy (2018) ao dizerem que a aprendizagem é um processo diferente entre cada aluno, sendo imprescindível, o uso de diferentes estratégias, para que o máximo de alunos consiga compreender os conteúdos abordados.

Foi observado pelo professor que a música e a pontuação apresentada a cada questão faz com que a plataforma se torne mais estimulante. Confirmando o estudo de Wang (2015) que avaliou a concentração, o aproveitamento, o engajamento e a diversão dos alunos jogando Kahoot utilizando ou não o áudio e a pontuação, sendo que quando não utilizou áudio e pontuação, os alunos não mantiveram o foco e a concentração da mesma forma. A apresentação da pontuação em cada questão fez com que os alunos se mantivessem mais concentrados e engajados, aumentando assim a competividade. Mostrando que a música e pontos, podem influenciar de maneira significativa no aprendizado. 
As respostas dos alunos confirmaram o trabalho de Santos e Santos (2017) que também apresentou como resultado a percepção de que os estudantes ficaram mais atentos e mais interessados nas aulas quando da aplicação do Kahoot Segundo Costa et. al. (2017), a participação dos estudantes em uma atividade gamificada do tipo quiz virtual como o kahoot favoreceu o aprendizado dos alunos. Concordando com Alves et. al. (2015), que a plataforma também contribuiu na construção do conhecimento, por despertar o interesse do aluno em acertar cada uma das questões e querer sempre ultrapassar os que estão nas melhores posições.

\section{ConClusão}

Por meio da realização deste trabalho, foi possível observar que a utilização da plataforma kahoot em sala de aula proporcionou uma maior e melhor interação entre aluno/professor e aluno/aluno, transformando o professor em um mediador do aprendizado. Com isso, além de auxiliar no processo de ensino-aprendizagem, percebe-se que a plataforma, quando bem utilizado pelo professor, contribui para a formação social e moral do indivíduo, principalmente quando trabalhado em grupo, despertando nos individuos o respeito entre os colegas. A utilização do kahoot possibilitou a obtenção de resultados significativos no ensino, provocou nos alunos um sentimento de desafio, gerando mais interesse e prazer pela aprendizagem. Para a continuidade da pesquisa pretende-se ampliar o numero de turmas e alunos de modo a melhorar a amostra e confirmar os resultados obtidos.

\section{REFERÊNCIAS}

Alves, R.M.M.; Geglio, P.C.; Moita, F.M.G.S.C.; Souza, C.N.S.; Araújo, M.S.M., (2015). O Quiz como Recurso Pedagógico no Processo Educacional: Apresentação de um Objeto de Aprendizagem. Anais do XIII Congresso Internacional de Tecnologia na Educação.

Amico, M. R. De A., Pra, R., Moraes, J. P. (2017). As aplicações do Kahoot! como tecnologia educativa. In: $22^{\circ}$ Seminário de Educação, Tecnologia e Sociedade, 2017, Taquara, RS. Anais Eletrônicos da Revista Educacional Interdisciplinar (REDIN).

Bottentuit Junior, J. B. (2012). Do computador aos tablets: vantagens pedagógicas na utilização de dispositivos móveis na educação. Revista Educa Online, 6(1):125-149.

Brown, R. B. (2006). Doing your Dissertation in Business and Management: The Reality of Researching and Writing. SAGE, London.

Costa, C. H. C.; Dantas Filho, F. F.; Gonçalves, Da S. C. M. F. M. (2017). Marvinsketch E Kahoot Como Ferramentas no Ensino de Isomeria. Holos (Natal. Online), v. 1, p. 31-43.

Covos, J. S.; Covos, J. F.; Rodrigues, F. R.; Ouchi, J. D. (2018). O Novo Perfil de Alunos no Ensino Superior, e a Utilização de Jogos Lúdicos para Facilitação do Ensino Aprendizagem. Educação em Foco (Amparo), v. 1, p. 62-74.

Dellos, R. (2015). Kahoot! A digital game resource for learning. International Journal of Instructional Technology and Distance Learning, 12(4), 49-52.

Gazotti-Vallim, M. A. (2017). Vivenciando inglês com Kahoot. The ESPecialist: Descrição, Ensino e Aprendizagem, 38(1):1-18.

Gómez, A. (2015). Educação na era digital - a escola educativa. Porto Alegre: Penso.

Izeki, C. A.; N., Walter A.; Dias, R. M. C. (2016). "Experiência no Uso de Ferramentas Online Gamificadas na Introdução à Programação de Computadores". Anais do XXII Workshop de Informática na Escola (WIE 2016), p. 301-310.

Kenski, V. M. (2012). Educação e tecnologias: o novo ritmo da informação. 8. ed. Campinas, SP: Papirus. 
Macedo, L. de, Petty, A. L. S, Passos, N. C. (2000). Aprender com jogos e situações problema. Porto Alegre: Artmed.

Prensky, M. (2012). Aprendizagem baseada em jogos digitais. São Paulo: Senac.

Sandy, D.; Sandy, D. (2018). Uso do Kahoot como Ferramenta de Avaliação e Ensino-Aprendizagem no Ensino de Microbiologia Industrial. Holos (Natal. Online), V. 1, P. 170-179.

Santos, D. S.; Santos, D. S. (2017). O QUIZ DO BIS: uso do kahoot como ferramenta de aprendizagem. In: III Congresso de Inovação e Metodologias no Ensino Superior, 2017, Belo Horizonte. Congresso de Inovação e Metodologias no Ensino Superior - CIM. v. 3.

Singh, K. (2007). Quantitative Social Research Methods. SAGE Publications, New Delhi.

Wang, A. I. (2015). The wear out effect of a game-based student response system. Computers and education. vol. 82. 